\title{
Normal Range of CD4 Cell Counts and Temporal Changes in Two HIV- Negative Malawian Populations
}

\author{
A.C. Crampin*, F.D. Mwaungulu, L.R. Ambrose, H. Longwe and N. French
}

Karonga Prevention Study, Malawi London School of Hygiene and Tropical Medicine, UK

\begin{abstract}
Longitudinal studies were carried out to determine trends in CD4 cell counts over a four year period in healthy HIV-negative adults in a rural (134 individuals) and an urban (80 individuals) site in Malawi, using TruCount ${ }^{\mathrm{TM}}$ and FACScount ${ }^{\mathrm{TM}}$ platforms. At baseline, median counts and $95 \%$ ranges were 890 (359-1954) cells per microlitre $(\mu 1)$ and 725 (114-1074) cells/ $\mu 1$ respectively. $1.5 \%$ and $6 \%$ respectively had baseline counts below 350 cells/ $\mu 1$ and $1.5 \%$ and $2.5 \%$ below 250 cells per $\mu \mathrm{l}$. Transient dips to below 250 cells $/ \mu 1$ were observed in seven individuals, with two individuals having persistently low CD4 counts over more than one year. Women and individuals from the urban site were significantly more likely to have "low CD4 count" $(<500$ cells $/ \mu 1)$ even when adjusted for other factors. In common with neighbouring countries, HIV-negative populations in Malawi have CD4 counts considerably lower than European reference ranges, and healthy individuals may have persistently or transiently low counts. Within Malawi, ranges differ according to the selected population.
\end{abstract}

Keywords: Malawi, CD4 count, HIV-negative, reference range.

\section{INTRODUCTION}

CD4+ lymphocyte (CD4) counts are routinely used world-wide, including many parts of sub-Saharan Africa, to determine eligibility for, and monitoring response to, antiretroviral therapy (ART) in HIV-positive patients. They are used as a measure of the risk of development of opportunistic infections. Well-established reference ranges from European and North American populations have proved inappropriate in some African populations in which the range of CD4 counts in apparently healthy HIV-negative individuals is significantly lower [1-5], although this finding is not replicated in all settings [2, 6, 7]. Less than 350 cells $/ \mu 1$ is considered a threshold for starting ART in HIVpositive patients without other indications in many highincome countries $[8,9]$ although not all $[10]$ and some commentators argue that with newer, safer antiretrovirals, there should be commencement of therapy at a higher threshold of CD4 count [11]. Similar levels of cut-off are encouraged for low-income countries where feasible [12] despite some evidence that treatment may be delayed further without increasing the risk of AIDS related events [13, 14]. There is consensus that, in the absence of clinical eligibility, it is critical to have a threshold of CD4 count for starting ART that ensures a favourable risk-benefit ratio at both individual and programme level. In sub-Saharan Africa, the WHO recommend starting ART in Stage III disease if the CD4 count is $<350$ cells/ $\mu 1$ or if the CD4 count is $<200$ cells $/ \mu 1$ irrespective of clinical stage [15]. In Malawi, eligibility for ART is usually determined by clinical criteria (Stage III or IV disease), but ART is made available to stage II or otherwise asymptomatic individuals with CD4 counts less than 250 cells $/ \mu 1$.

*Address correspondence to this author at the Karonga Prevention Study, Malawi London School of Hygiene and Tropical Medicine, UK; Tel: + 265 999373 980; E-mail: mia.crampin@1shtm.ac.uk
In settings where HIV-negative individuals (and therefore presumably relatively immunocompetent HIVpositive individuals) are frequently found to have CD4 counts below <350cells $/ \mu$ l (up to $5 \%$ of healthy HIV negative adults) [2], it is frequently stated that there is considerable intra-patient variability and that CD4 count may be depressed by acute infections [16, 17]. Some published reports on CD4 trends in HIV positive individuals describe the variability of CD4 count over time in healthy HIV-negative individuals in sub-Saharan Africa but data are not presented on whether the extremes that overlap with eligibility for ART are transient or persistent effects $[18,19]$. It is likely that whatever variability is observed, that CD4 counts will vary more in HIV-positive individuals even if clinically stable $[20,21]$, however, an understanding of the "normal" variability in uninfected individuals is key to interpretation of clinical thresholds, reference ranges and mathematical models predicting risk of opportunistic diseases and impact of HIV [2].

As part of two immunological studies in Malawi, one of contacts of tuberculosis cases and one of safety and immunogenicity of pneumococcal vaccines we performed sequential CD4 counts on persistently HIV-negative adults over a four year period.

\section{MATERIALS AND METHODS}

HIV-negative spouses of smear positive tuberculosis patients consented to recruitment for a follow-up study of $M$. tuberculosis infection and disease rates, in Karonga, a rural district of Malawi. At baseline, 6, 12, 24 and 36 months, individuals were counselled and tested for HIV testing and had CD4 counts performed using flow cytometry. Initial HIV-antibody testing of plasma was performed using an ELISA (Organon Vironostika) and particle agglutination test (Edgware modification of the Serodia). Samples giving discrepant results were repeated in duplicate using the same 
two tests. EDTA-anticoagulated whole blood was labelled with the TriTEST ${ }^{\mathrm{TM}}$ CD3 FITC/CD4 PE/CD45 PerCP monoclonal antibody mix in TruCount ${ }^{\mathrm{TM}}$ tubes to determine absolute cell counts. Samples were acquired on a FACSCalibur $^{\mathrm{TM}}$ and analysed using MultiSET ${ }^{\mathrm{TM}}$ software. All reagents, hardware and software were purchased from Becton Dickinson (UK) and used according to manufacturer's instructions.

Healthy HIV-negative individuals consented to recruitment as controls in a study of immune response to pneumococcal conjugate vaccine in Blantyre, a large city in Malawi. Participants came forward as volunteers following a public advert. CD4 counts were measured at baseline and one month following each of two doses of conjugate pneumococcal vaccine then subsequently every six to a maximum of 48 months. The HIV status of participants was measured using a twin EIA method (Vironostika HIV uniForm II plus O, Biomerieux, Boxtel, Netherlands and Murex HIV-1.2.0, Dartford, UK). Discrepant results were checked using two rapid tests (Determine HIV-1/2/O, Abbott Laboratories, Abbott and Uni-Gold Recombinant HIV-1/2 Trinity Biotech,). CD4 counts were measured using a FACScount $^{\mathrm{TM}}$ (Becton Dickinson) system according to the manufacturer's instructions. The Laboratory participated in the UK's national external quality assurance programme for CD4 count measurement; during the study period 25 CD4 count measurements were returned. On no occasion was a result returned that was below the trimmed mean, with 21 of the 25 within $+1 \mathrm{SD}$ of trimmed mean. HIV-status was assessed throughout the period of follow-up by sequential testing. Individuals who HIV-seroconverted during either study were excluded from this analysis at the last time-point at which they were HIV-antibody negative.

Ranges of CD4 count, with 95\% ranges, means and standard deviations are given, and the chi squared test used to compare grouped indices by site.

The rural study was approved by the National Health Science Research Committee of Malawi and the London School of Hygiene ethical review board. The urban study was approved by the Malawi College of Medicine Research Ethics Committee and the Liverpool School of Tropical
Medicine, institutional review board.

\section{RESULTS}

In Karonga 134 individuals were recruited and had CD4 counts performed. In 72 individuals CD4 counts were available at two time points, in 52 at three time points and in 20 at four time points. In the urban Blantyre population, 80 individuals were recruited; 75 had sampling at two time points, 59 at three and 53 at four or more, to a maximum of 16. Approximately half of the participants had received the study vaccine.

The Karonga population was older (mean age 42 years, compared to 33 years) and a higher proportion were females $(63 \%$ to $29 \%)$ than in Blantyre.

The distribution of total lymphocyte count was similar between the two sites when all samples with accompanying haematological indices were considered (Blantyre range 0.36 to 5.0, mean 1.91, standard deviation 0.734, with two excluded outliers over $20, \mathrm{n}=432$, Karonga range 0.47 to 4.68, mean 2.08, sd 0.733, $\mathrm{n}=258$ ).

Samples in Karonga were processed a mean of 3.4 hours after collection (range 0 to 8 hours). Samples in Blantyre were processed on the day of collection i.e. within six hours maximum, but times for individual samples were not recorded.

The ranges of CD4 counts differed between the sites, both at baseline and when all results from repeated samplings were considered together. Summary statistics on individuals at the urban site were lower than at the rural site in both males and females (Table 1). The crude risk of "low CD4 count" using an international range $(<500$ cells $/ \mu \mathrm{l})$ was significantly higher in Blantyre than Karonga $(24 \%$ compared to $9 \%, \quad$ chi $=8.85, \quad \mathrm{p}=0.003$ ) and remained significantly different when adjusted for age and sex $(20 \% \mathrm{vs}$ $7 \%$, chi=7.38, $\mathrm{p}=0.006)$. Data on 59 HIV-negative individuals from a previous community cross-sectional survey in Karonga are also presented [22]. Low CD4 count was associated with lymphopenia at both sites.

The distribution of all CD4 counts by site is displayed in Fig. (1).

Table 1. Range and Distribution of CD4 Cell Counts in Karonga and Blantyre

\begin{tabular}{|c|c|c|c|c|c|c|c|}
\hline & \multicolumn{7}{|c|}{ Baseline } \\
\hline & $\mathbf{n}$ & Range & 95\% Range & Mean (sd) & Median & $<350$ Cells $/ \mu \mathrm{l}$ & $<250$ Cells $/ \mu l$ \\
\hline Karonga (rural) & 134 & $208-2335$ & $359-1954$ & 949 (364) & 890 & $1.5 \%$ & $1.5 \%$ \\
\hline Male & 49 & $356-1753$ & $376-1680$ & $921(289)$ & 876 & & \\
\hline Female & 85 & $208-2335$ & $255-2206$ & $964(402)$ & 938 & & \\
\hline Blantyre (urban) & 80 & $114-1736$ & $114-1074$ & $720(281)$ & 725 & $6 \%$ & $2.5 \%$ \\
\hline Male & 57 & $114-1736$ & $114-1144$ & 702 (287) & 669 & & \\
\hline Female & 23 & $307-1315$ & $307-1315$ & $763(269)$ & 809 & & \\
\hline All & 214 & $114-2335$ & $276-1730$ & $863(353)$ & 836 & $3 \%$ & $2 \%$ \\
\hline Karonga Survey & 59 & $130-1654$ & $199-1585$ & 922 (348) & 892 & $5 \%$ & $1.5 \%$ \\
\hline
\end{tabular}




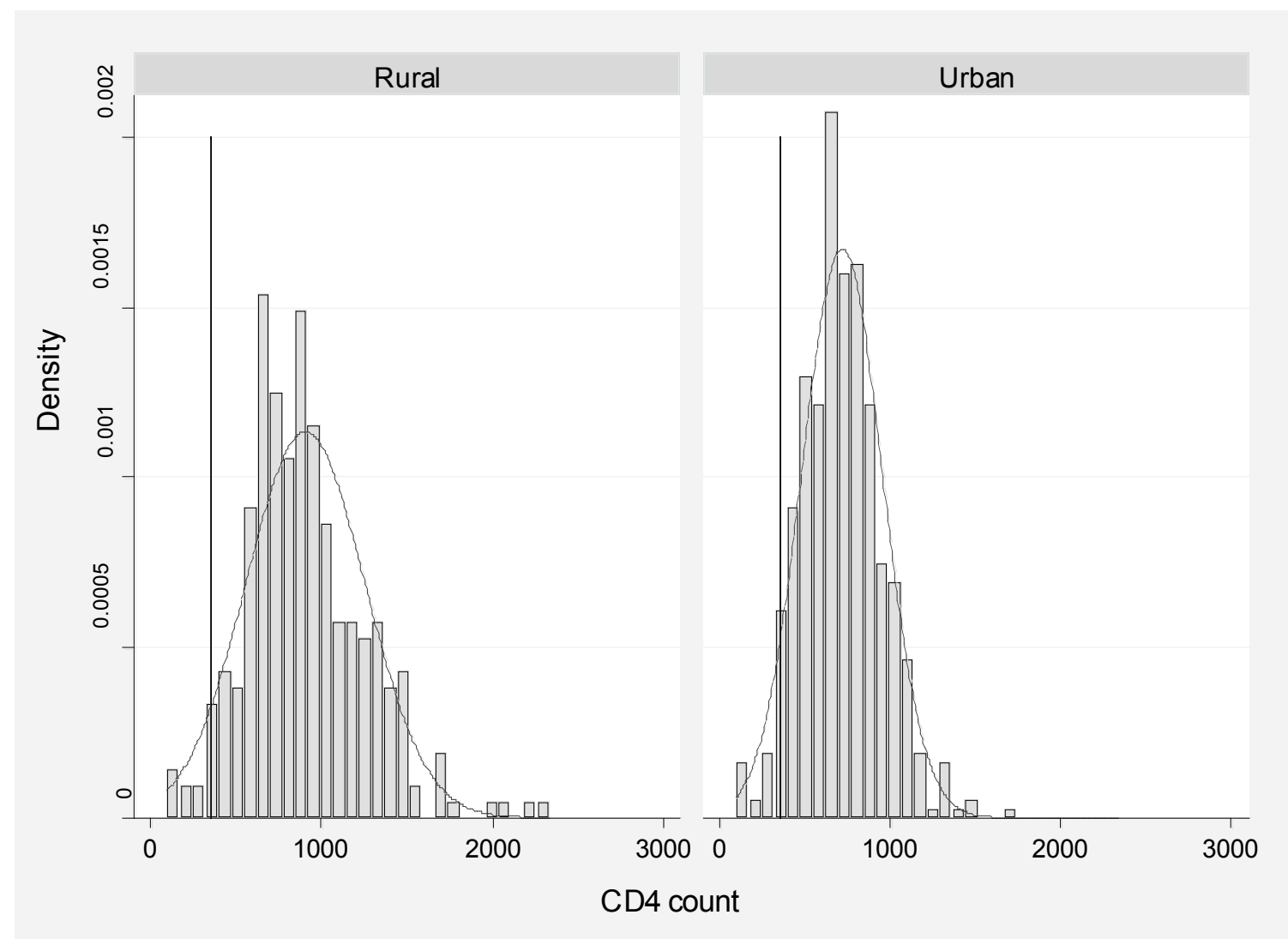

Fig. (1). Distribution of all CD4 counts by site of study.

For the purposes of examining trends in CD4, combined urban/rural baseline results were grouped into "bands" of 0250 (Very low), 251-350 (Low), 351-500 (Lower normal) and $>500$ cells $/ \mu$ (Higher normal). Of those with follow-up, $108 / 151(72 \%)$ of individuals remained in the same band for the duration of followup, 1/3 Very low, 0/3 Low, 3/22 Low normal, 104/123 Normal.

24/151 moved to a higher band (some transiently) and $24 / 151$ to a lower band (some transiently). A transient dip to 239 cells $/ \mu$ in one individual who otherwise consistently had CD4 counts $>500$ cells $/ \mu$ was known to coincide with a non-malarial, non-bacteraemic acute febrile illness. The detail for each banding is given in Table 2 .

To give a more detailed picture of the stability of CD4 count in a sample of the group, trends over four years for women aged 20-29 with at least one year of follow-up are presented graphically in Fig. (2). These graphs include two individuals, highlighted in Fig. (2), from the rural population, who had profoundly low CD4 counts $(<250$ cells $/ \mu \mathrm{l})$ but were clinically well throughout the study and were persistently HIV-negative. On 2-year follow-up after the conclusion of the study, one had died suddenly with haematemesis (suggesting occult abdominal disease, despite regular clinical follow-up) and the other remains clinically well, although all haematological indices remain slightly low, suggesting the low CD4 count was part of a general picture rather than a specific deficit. The figure also includes two individuals (also highlighted) with transient "dips" to below 250 cells $/ \mu 1$.

\section{DISCUSSION}

The ranges of CD4 counts seen in these studies are similar to those from studies in Ethiopia, Central African Republic, Guinea Bissau and Botswana (Table 3) and are significantly lower than the those found in European populations [1]. In common with the other populations with lower mean counts, $3 \%$ of this Malawian population had a

Table 2. Change in CD4 Cell Count Band Over Course of Study

\begin{tabular}{|c|c|c|c|}
\hline Initial CD4 Count (Cells/ $\boldsymbol{\mu l})$ & Moved to Lower Band & Same Band Throughout & Moved to Higher Band \\
\hline \hline Very low <=250 & n/a & 1 & 3 \\
\hline Low 250-350 & 0 & 0 & 3 \\
\hline Lower normal 350-500 & 5 & 3 & 18 \\
\hline Higher normal 500+ & 19 & 104 & 22 \\
\hline Total & 24 & 108 & 123 \\
\hline
\end{tabular}




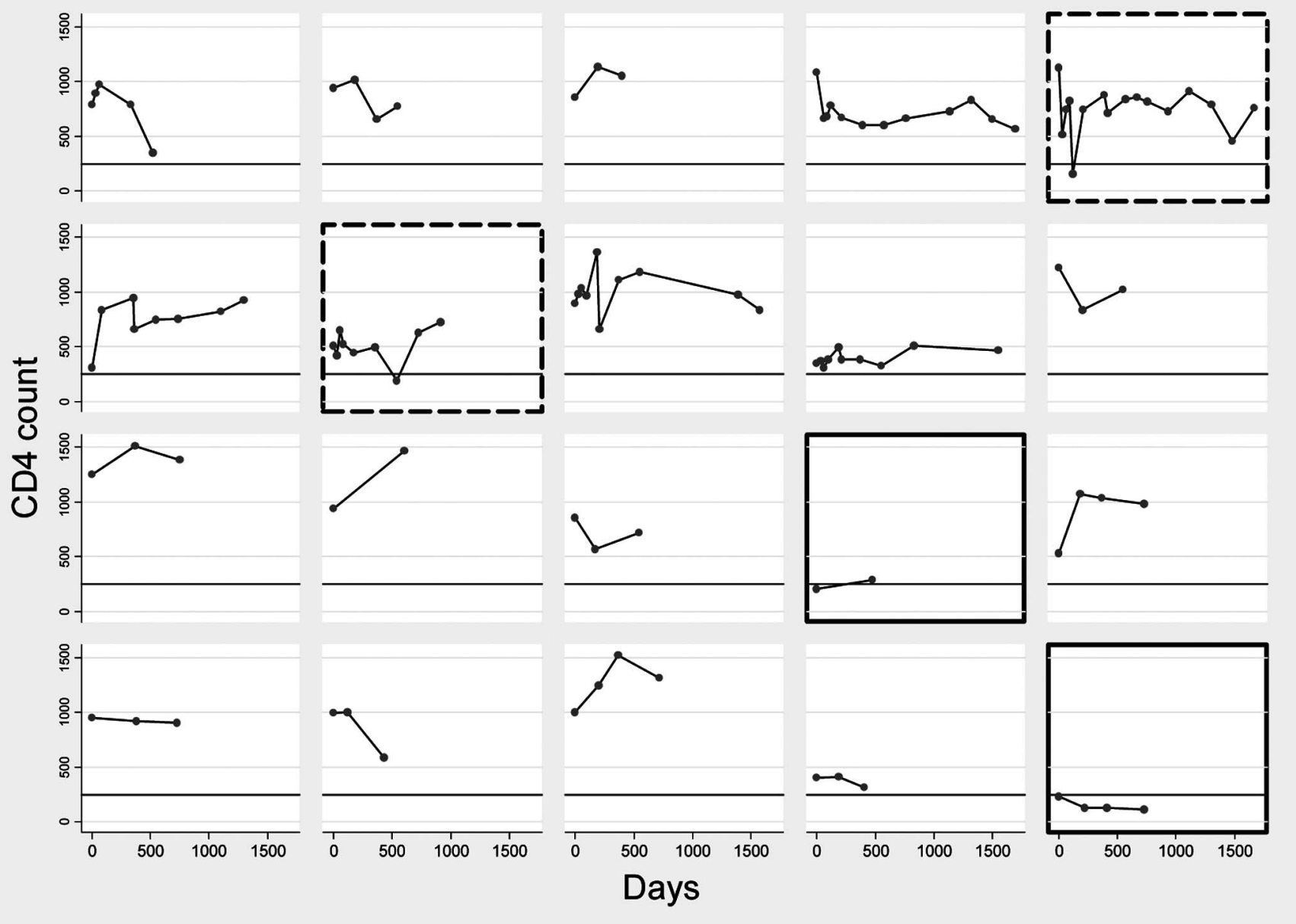

Fig. (2). Details of trends of CD4 count over 4 years in a sample of 20 women aged 20-39 years by individual. Line at 250 cells/ $\mu 1$.

Footnote: Two individuals with persistently low CD4 counts are highlighted and two with transient dips - - - - - - .

CD4 count less than 350 cells $/ \mu$ on a single sampling, and $9 \%$ had a low CD4 count at some point when sampled repeatedly over a 3 year period. In this study, each time the individuals were sampled, the HIV test was repeated so the low CD4 counts are known not to be related to false-negative HIV tests, as might occur in single-sampling studies.

In nine individuals a CD4 count was recorded below a threshold determining eligibility for ART in HIV-positive patients according to Malawi ART programme guidelines (250 cells/ $\mu$ l). In seven of these people (all from Blantyre), the low CD4 count was an isolated event restored to a "normal" level one month later. In two (both from Karonga), this was part of a persistently low CD4 count over more than one year. All the transient dips were found in the urban site, and although one was known to coincide with an acute febrile illness, assay-related effects cannot be discounted. The differences may, however, be a function of the increased frequency of sampling in the urban population, poorer health or other psychological or biological urban factors. Several of these individuals are represented in Fig. (2).

Extremely low CD4 counts are observed in some apparently healthy HIV-negative subjects in Africa. When this is persistent, as with two individuals in the rural study, it is likely to represent occult disease of some severity or a chronic haemapoetic disorder. In others this may represent the end of a normal distribution, however low counts in apparently healthy individuals are worthy of investigation.

We observed clear and significant differences between the sites. This may be inherent to the population, due to the selection processes for the different studies or an artefact of the different laboratory methods for estimation of CD4 count. It is notable that total lymphocyte counts had similar distributions in the two sites. Several North American studies have demonstrated consistent inter-laboratory variability in measurements despite shared protocols and participation in quality assurance programmes [23-25]. Due to the distance between laboratories we were unable to conduct comparability experiments on shared samples although comparison studies at one of the sites found no systematic variation in results between the two platforms even at low CD4 counts [26] as has been demonstrated in similar settings [27] and UK's national external quality assurance programme shows no difference in mean CD4 count returns. It is possible that the recent exposure of the rural group to tuberculosis might have had an effect on CD4 count as it is known that active disease may depress the level [28]. However none of the individuals in this group progressed to clinical tuberculosis and the range was actually 
Table 3. Comparison of CD4 Ranges in HIV Negative Individuals from Sub-Saharan African Countries, Ordered by Mean CD4 Count

\begin{tabular}{|c|c|c|c|c|c|}
\hline \multirow{2}{*}{ Country } & \multirow{2}{*}{ Population Sampled } & \multicolumn{4}{|c|}{ CD4 Count } \\
\cline { 3 - 6 } & & N & Mean (sd) & Median & 95\% Range \\
\hline \hline Ethiopia [1] & Healthy adults & 142 & $775+/-225$ & 761 & $366-1235$ \\
\hline Nigeria [21] & Miners (M+F) & 183 & $828+/-203$ & $547-1327$ \\
\hline Malawi & see above & 214 & $863+/-353$ & 836 & $276-1730$ \\
\hline Guinea Bissau [5] & Healthy adults & 133 & $892+/-107$ & 819 & 851 \\
\hline Central African Republic [3] & Healthy men & M 68 & $927+/-349$ & $380-1617$ \\
\hline Tanzania [6] & Healthy adults & 147 & $968+/-$ & 980 & $386-1454$ \\
\hline Uganda [7] & & 183 & $1256+/-$ & 1160 & \\
\hline
\end{tabular}

slightly higher than that seen in the urban group. There was no suggestion from the data that the administration of the conjugate pneumococcal vaccine was suppressing the CD4 count as baseline counts would have been unaffected. Although the differences between our two sites were not so great that different conclusions to the study would be drawn, the differences highlight that sampling a single population within a country may not give an accurate reference range for country-wide application, and that any reference range may be dependent on the platform used for CD4 counting.

The higher values seen in the women in our studies are consistent with findings elsewhere [29, 30], and as found in another study [30] we also observed a decrease with age (not presented) although this was not significant.

Diurnal variation in CD4 count is known to occur in both HIV-negative and HIV-positive individuals, with counts increasing during the day particularly in those who are HIVnegative [31]. In both studies, where time of venepuncture was recorded, $97 \%$ of samples were collected between a four hour morning period, so timing is unlikely to account for any intra-patient variability.

Overall CD4 count may be a less useful marker of prognosis in AIDS care in sub-Saharan Africa than elsewhere [32], but given the investment in CD4 infrastructure, it is important to ensure that its usefulness is optimised locally. Current Malawi ART programme guidelines define eligibility for ART on clinical grounds (WHO stage III or IV disease) or with a CD4 count $<250$ cells $/ \mu 1$, and CD4 counts are becoming more widely available. The studies presented here provide population data against which CD4 counts in HIV-positive individuals may be evaluated and provide insight into the natural variation in CD4 counts both between and within individuals in this setting.

\section{CONFLICT OF INTEREST}

None.

\section{ACKNOWLEDGEMENTS}

This paper is dedicated to the late Frank Mwaungulu who designed and conducted the rural study and who died in a tragic accident before completion of the study. The studies were supported by the Wellcome Trust and the British Leprosy Relief Association.

\section{ABBREVIATIONS}

HIV $=$ Human immunodeficiency virus

$\mu \mathrm{l}=$ Microlitre

WHO $=$ World Health Organisation

ART = Anti Retroviral Therapy

\section{REFERENCES}

[1] Tsegaye A, Messele T, Tilahun $\mathrm{T}$, et al. Immunohematological reference ranges for adult Ethiopians. Clin Diagn Lab Immunol 1999; 6(3): 410-4

[2] Williams BG, Korenromp EL, Gouws E, Schmid GP, Auvert B, Dye C. HIV infection, antiretroviral therapy, and CD4+ cell count distributions in African populations. J Infect Dis 2006; 194(10): 1450-8.

[3] Menard D, Mandeng MJ, Tothy MB, Kelembho EK, Gresenguet G, Talarmin A. Immunohematological reference ranges for adults from the Central African Republic. Clin Diagn Lab Immunol 2003; 10(3): 443-5.

[4] Bussmann H, Wester CW, Masupu KV, et al. Low CD4+ Tlymphocyte values in human immunodeficiency virus-negative adults in Botswana. Clin Diagn Lab Immunol 2004; 11(5): 930-5.

[5] Ricard D, Wilkins A, N'Gum PT, et al. The effects of HIV-2 infection in a rural area of Guinea-Bissau. AIDS 1994; 8(7): 97782.

[6] Levin A, Brubaker G, Shao JS, et al. Determination of Tlymphocyte subsets on site in rural Tanzania: results in HIV-1 infected and non-infected individuals. Int J STD AIDS 1996; 7(4): 288-91.

[7] Tugume SB, Piwowar EM, Lutalo T, et al. Hematological reference ranges among healthy Ugandans. Clin Diagn Lab Immunol 1995; 2(2): 233-5.

[8] Panel on Antiretroviral Guidelines for Adults and Adolescents Guidelines for the use of antiretroviral agents in HIV-1-infected adults and adolescents. Department of Health and Human Services. January 29, 2008; 1-128. Available at http://www.aidsinfo.nih.gov/ ContentFiles/AdultandAdolescentGL.pdf [Accessed 01/05/2008 (p12)].

[9] British HIV Association guidelines for treatment of HIV-infected adults with antiretroviral therapy. 2008. http://www.bhiva.org/files/ file1030835.pdf [Accessed 01/05/2008 (p11)].

[10] Josephson F, Albert J, Flamholc L, et al. Antiretroviral treatment of HIV infection: Swedish recommendations 2007. Scand J Infect Dis 2007; 39(6-7): 486-507.

[11] Gallant JE. Should antiretroviral therapy be started earlier? Curr HIV/AIDS Rep 2007; 4(2): 53-9.

[12] Antiretroviral therapy for HIV infection in adults and adolescents: Recommendations for a public health approach. 2006 revision, 
http://www.who.int/hiv/pub/guidelines/artadultguidelines.pdf [Accessed 01/05/2008 (p14)].

[13] Wood E, Hogg RS, Yip B, Harrigan PR, O'Shaughnessy MV, Montaner JS. Is there a baseline CD4 cell count that precludes a survival response to modern antiretroviral therapy? AIDS 2003; 17(5): 711-20

[14] Ahdieh-Grant L, Yamashita TE, Phair JP, et al. When to initiate highly active antiretroviral therapy: a cohort approach. Am J Epidemiol 2003; 157(8): 738-46.

[15] (WHO) WHO. Interim WHO clinical staging of HIV/AIDS and HIV/AIDS case definitions for surveillance: African region. Geneva 2005.

[16] Anzala AO, Simonsen JN, Kimani J, et al. Acute sexually transmitted infections increase human immunodeficiency virus type 1 plasma viremia, increase plasma type 2 cytokines, and decrease CD4 cell counts. J Infect Dis 2000; 182(2): 459-66.

[17] Chirenda J. Low CD4 count in HIV negative malaria cases and normal CD4 count in HIV positive and malaria negative patients. Cent Afr J Med 1999; 45(9): 248.

[18] Mboto CI, Davies-Russell A, Fielder M, Jewell AP. CD4+ lymphocyte values and trends in individuals infected with Human Immunodeficiency Virus and/or co-infected with Hepatitis C Virus in The Gambia. Afr Health Sci 2009; 9(3): 130-6.

[19] Urassa W, Bakari M, Sandstrom E, et al. Rate of decline of absolute number and percentage of CD4 lymphocytes among HIV1 infected adults in Dar es Salaam, Tanzania. AIDS 2004; 18(3): 433-8.

[20] Raboud JM, Haley L, Montaner JS, Murphy C, Januszewska M, Schechter MT. Quantification of the variation due to laboratory and physiologic sources in CD4 lymphocyte counts of clinically stable HIV-infected individuals. J Acquir Immune Defic Syndr Hum Retrovirol 1995; 10(Suppl 2): S67-73.

[21] Taylor JM, Tan SJ, Detels R, Giorgi JV. Applications of a computer simulation model of the natural history of CD4 T-cell number in HIV-infected individuals. AIDS 1991; 5(2): 159-67.

[22] McGrath N, Kranzer K, Saul J, et al. Estimating the need for antiretroviral treatment and an assessment of a simplified
HIV/AIDS case definition in rural Malawi. AIDS 2007; 21(Suppl 6): $\mathrm{S} 105-13$.

[23] Sax PE, Boswell SL, White-Guthro M, Hirsch MS. Potential clinical implications of interlaboratory variability in CD4+ Tlymphocyte counts of patients infected with human immunodeficiency virus. Clin Infect Dis 1995; 21(5): 1121-5.

[24] Mandigo K, Raboud JM, Haley L, Montaner JS. Variability in leukocyte subset measurements among five laboratories in Vancouver. Clin Invest Med 1995; 18(5): 349-56.

[25] Fei DT, Paxton H, Chen AB. Difficulties in precise quantitation of CD4+ T lymphocytes for clinical trials: a review. Biologicals 1993; 21(3): 221-31.

[26] MacLennan CA, Liu MK, White SA, et al. Diagnostic accuracy and clinical utility of a simplified low cost method of counting CD4 cells with flow cytometry in Malawi: diagnostic accuracy study. BMJ 2007; 335(7612): 190 .

[27] Dieye TN, Vereecken C, Diallo AA, et al. Absolute CD4 T-cell counting in resource-poor settings: direct volumetric measurements versus bead-based clinical flow cytometry instruments. J Acquir Immune Defic Syndr 2005; 39(1): 32-7.

[28] Turett GS, Telzak EE. Normalization of CD4+ T-lymphocyte depletion in patients without HIV infection treated for tuberculosis Chest 1994; 105(5): 1335-7.

[29] Prins M, Robertson JR, Brettle RP, et al. Do gender differences in CD4 cell counts matter? AIDS 1999; 13(17): 2361-4.

[30] Lugada ES, Mermin J, Kaharuza F, et al. Population-based hematologic and immunologic reference values for a healthy Ugandan population. Clin Diagn Lab Immunol 2004; 11(1): 29-34.

[31] Malone JL, Simms TE, Gray GC, Wagner KF, Burge JR, Burke DS. Sources of variability in repeated T-helper lymphocyte counts from human immunodeficiency virus type 1-infected patients: total lymphocyte count fluctuations and diurnal cycle are important. J Acquir Immune Defic Syndr 1990; 3(2): 144-51.

[32] Badri M, Bekker LG, Orrell C, Pitt J, Cilliers F, Wood R. Initiating highly active antiretroviral therapy in sub-Saharan Africa: an assessment of the revised World Health Organization scaling-up guidelines. AIDS 2004; 18(8): 1159-68.

(C) Crampin et al.; Licensee Bentham Open.

This is an open access article licensed under the terms of the Creative Commons Attribution Non-Commercial License (http://creativecommons.org/licenses/by-nc/ $3.0 /$ ) which permits unrestricted, non-commercial use, distribution and reproduction in any medium, provided the work is properly cited. 